\section{Making the Grade Choosing the Right CCD Camera Chip Grade}

Ted Inoue, Video Microscopy, Inc.

\section{Introduction}

Today, charge coupled devices (CCDs) have become the de-facto standard for scientific imaging. In fact, their potential for high resolution, sensitivity, wide dynamic range and low noise has made them the device of choice for most fluorescent microscopy imaging applications. This is especially true for quantitative microscopy applications such as ratio imaging, fluorescent in-situ hybridization and image deconvolution.

However, choosing the most appropriate CCD camera for each application can be quite challenging given the broad range of features and performance specifications. This month's article focuses on a single characteristic of the CCD: the chip grade.

The grade of a CCD sensor is, unfortunately, one of its least discussed characteristics. Often, manufacturers and salespeople avoid talking about chip grade as they see it as a measure of how far a chip is from perfection. Hence, to discuss chip grade implies camera defects. Defects that can lead to black or white spots or even lines across the image! For camera vendors, it is a sore point because they know their users would be most satisfied if they could provide perfect chips to everyone. Likewise, it is a point of contention for users who feel that camera vendors are somehow passing off defective merchandise. Fortunately, the solution is simple: be an educated consumer and choose the most appropriate chip grade for one's primary applications.

\section{Why Aren't All Chips Perfect?}

Charged Coupled Devices are large semiconductor devices, created in the same manner as other integrated circuits. Just a few years ago, it was considered an amazing feat when engineers fabricated the first milliontransistor chip. Amazing, because such a chip contains so many elements that the probability for errors to arise became close to $100 \%$.

In smaller chips with fewer elements, this is not such a problem for a number of reasons. First, one can produce small chips in very large quantities, allowing one to discard defects. In the semiconductor business, the ratio of good chips to defects is called the "chip yield". However, as chip size increases, the yield decreases dramatically. Hence large chips without defects are quite rare and therefore they are very expensive to produce.

Imagine the added difficulty of creating a "perfect" CCD array. Each pixel on a CCD is an individual area of the chip that is sensitive to light. If any pixel has a different response from its neighbors, it will read a different value. In normal computer chips, slight variations of this sort are acceptable because digital circuits accept a wide range of values as either "on" or "off". CCDs are quite different. Research grade cameras might be used to measure changes of only a few percent, hence a pixel that has a twenty percent deviation from the average would typically be considered defective.
To summarize - defects are inevitable in the current semiconductor manu-oे facturing process. The larger the device, the more likely it is to have defects.

\section{Why Sell Defective Chips?}

As noted, chip yield results in high prices for near perfect chips. Camera manufacturers could choose to sell only cameras with perfect chips, but these might cost more than most users could afford. Hence they sell "imperfect" chips at a discount. This seems a rational decision, as the defects are often minor: ao handful of random pixels might be abnormal or perhaps a column or two mighto be "dead". In addition, many of the problems can be fixed via software. The re-sult is that one can now purchase cameras using large, high resolution CCDs much more affordably than would be otherwise possible.

\section{What Types of Defects Exist?}

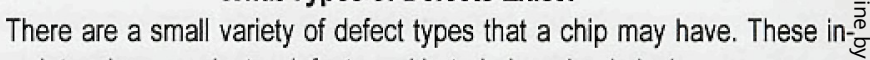
clude point, column or cluster defects and hot, dark or dead pixels.

Point defects are the most benign type of chip defect. A small number ofo pixels that do not respond exactly the same as their neighbors poses little threato. to accurate quantitation as they can be ignored or replaced based on their neigh-s. bors' values using a simple algorithm.

Point defects fall into the following sub-categories:

Dead pixels - (Figure 1) pixels that are totally non-responsive resulting in in pixels that always read some constant value.

Dark pixels - these are pixels that are less sensitive than they should be and hence return a low value

Hot pixels - (Figure 2) pixels that build up charge faster than they should, resulting in bright spots on the image.

Column defects (Figure 3 ) are typically entire columns of dead pixels. These are problematic, as they are visually distracting and are more difficult to remove than isolated pixel defects.

Cluster defects consist of more than one adjacent pixel containing defects. I've never seen cluster defects, and camera people only talk about them in literature. Therefore they're not included in the chip-grading list below, but clusters would be put in a better grade than line defects, perhaps grade 1 if there were a cluster or two. A handful of clusters might be considered as objectionable as one line defect. Unfortunately, manufacturers don't even seem to talk about cluster defects in their ratings!

The following list is a guide to the grades used by chip manufacturers to specify the kinds of defects that are allowable on a chip. By reviewing this and the accompanying pictures, you can gain an understanding of what to expect from a given camera chip grade. Note that different manufacturers may grade chips differently.

Grade 0: Perfect, as in zero defects. A perfect chip.

Grade 1: This chip may contain a small number of point defects but may not contain any column defects.

Grade 2: One or "a few" column defects and/or many point defects.

Grade 3: Many point and column defects

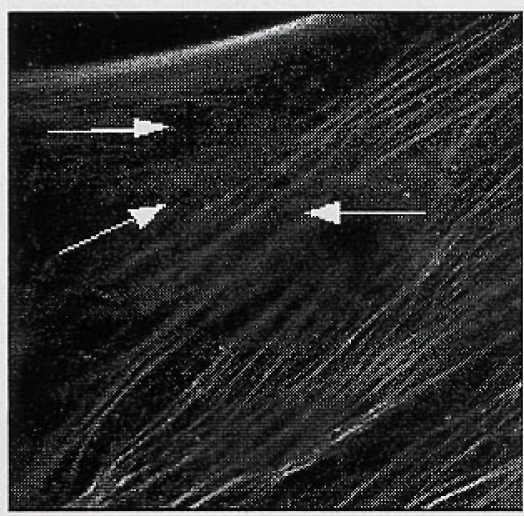

Figure 1: Dead pixels

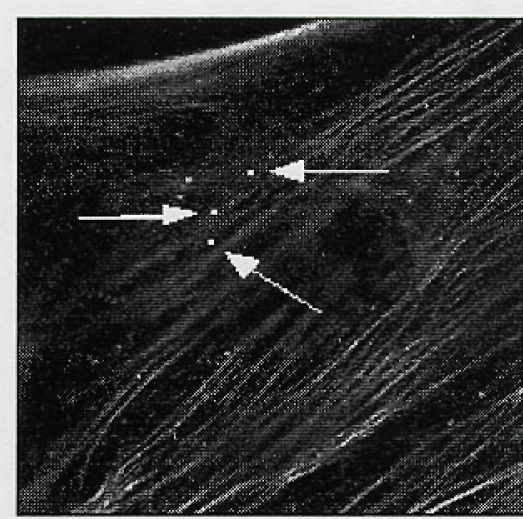

Figure 2: hot Pixels

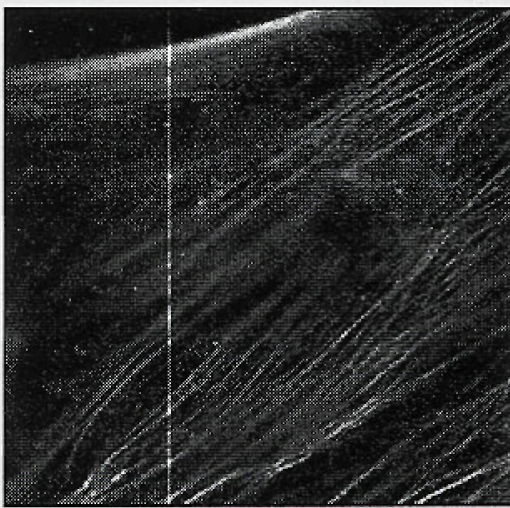

Figure 3: Column defect 
Discussion

The previous list roughly defines the chip grades, outlining basic chip defects that one can expect. Unfortunately, the manufacturers have chosen not to standardize their chip grading criteria, resulting in great confusion for consumers. Today, one manufacturer's grade 1 chip may be considerably different from another's, making it important to learn from the vendor exactly how they grade each chip.

Fortunately, chip quality is steadily improving, and the currently popular Sony interline transfer chips are rated by Sony as "perfect". So perhaps within the next few years, all cameras will give picture perfect results every time. But for now, look carefully and understand completely exactly what is being purchased.

References:

Cosmetic Grading for Kodak Sensors:

http://www. apogee-ccd.com/kodak_cosmetic.html

Cosmetic Grading for SITe Sensors:

http://www.apogee-ccd.com/site_cosmetic.htm

Selection of CCD Arrays:

http://www. prinst.com/pdfs/pg15.pdf

Application of Stack Filter in CMOS Imager Whitespot Compensation:

http://yake.ecn.purdue.edu/ jijen/Application/cmos.html

For a large number of WWW references to specific chip grading practices of the manufacturers, please see the VideoMicroscopy Web site:

http://www.videomicroscopy.com/Tutorials/CCD\%20Grading/ CCD\%20grades.htm
WinEDS 3.0 High Performance X-ray Microanalysis For Windows ${ }^{\mathrm{TM}} 95$ / NT 4.0

This FAST full 32-bit PC Windows ${ }^{\mathrm{TM}}$ based analyzer gives you all the performance of more expensive workstation systems.

* Qualitative \& Quantitative software with one click operation.

* WinEDS PX9000 Pulse Processor DX3000 Bias.

x Interfaces to several detector brands.

* 5 to $10 \mathrm{eV}$ improvement on most detector's resolutions.

* Complete system upgrades - including new detectors.

* Partial upgrades to suit your system needs. Also

× Complete line of PC based imaging systems.

* Top dollar for trade-ins.

* Service for TN5500 and TN2000 systems.

TN Analyzer Service Inc. 7897 Hwy 19

Dane, WI 53529

Tel.: (608)798-2005, Fax: (608)798-1675, eMail: doug_tnas@msn.com

Visit our web site: www.tnanalyzerservice.com

Now with demo labs in Florida and Texas, as well as in Wisconsin - and soon on the west coast.
WE SERVICE COMPUTER CONTROLLERS BEAM BLANKERS WATER CHILLERS
Contracts and On-Demand

Emergency Service at

Reasonable Rates from

Factory Trained Specialists.

1-800-421-8451
PREP EQUIPMENT

ULTRAMICROTOMES DUPONT \& RMC

VACUUM COATERS SPUTTER COATERS MECHANICAL PUMPS TURBO PUMPS PLASMA ASHERS 